\title{
Parametric Analysis of Flexible Logic Control Model
}

\author{
Lihua Fu, Dan Wang, and Jinyun Kuang \\ College of Computer Science, Beijing University of Technology, Beijing 100124, China \\ Correspondence should be addressed to Lihua Fu; fulihuapaper@sohu.com
}

Received 20 September 2012; Revised 26 December 2012; Accepted 1 January 2013

Academic Editor: Xiang Li

Copyright (C) 2013 Lihua Fu et al. This is an open access article distributed under the Creative Commons Attribution License, which permits unrestricted use, distribution, and reproduction in any medium, provided the original work is properly cited.

\begin{abstract}
Based on deep analysis about the essential relation between two input variables of normal two-dimensional fuzzy controller, we used universal combinatorial operation model to describe the logic relationship and gave a flexible logic control method to realize the effective control for complex system. In practical control application, how to determine the general correlation coefficient of flexible logic control model is a problem for further studies. First, the conventional universal combinatorial operation model has been limited in the interval $[0,1]$. Consequently, this paper studies a kind of universal combinatorial operation model based on the interval $[a, b]$. And some important theorems are given and proved, which provide a foundation for the flexible logic control method. For dealing reasonably with the complex relations of every factor in complex system, a kind of universal combinatorial operation model with unequal weights is put forward. Then, this paper has carried out the parametric analysis of flexible logic control model. And some research results have been given, which have important directive to determine the values of the general correlation coefficients in practical control application.
\end{abstract}

\section{Introduction}

Fuzzy control has made the rapid development, and it has found a considerable number of successful industrial applications in recent years [1]. However, from the mathematical viewpoint, Professor Li revealed the interpolation mechanism of fuzzy control and proved that fuzzy controller is in essence an interpolator [2]. So, there are two problems in controlling some practical complex systems. One is that control rules will grow exponentially with the growing of inputs, and the other one is that the precision of control system is not high [3].

Compound controllers combine fuzzy control and other relatively mature control methods to obtain effective control effect, such as Fuzzy-PID controllers [4], fuzzy prediction control [5], and adaptive fuzzy $H$-infinity control [6]. To reduce dimensionality, hierarchical fuzzy logic controller separates the set of control rules into several sets based on different functions $[7,8]$. The basic idea of adaptive fuzzy controllers based on variable universe is to keep the control rules unchanged and change the region bound of fuzzy variables with the values of input fuzzy variables in order to increase control rules indirectly [9]. Though a great deal of research has been done to improve the performance of fuzzy control, most of these methods are based on the basic idea that fuzzy controller is a piecewise approximation. However, to date, there has been relatively little research conducted on the internal relations among input variables of fuzzy controllers.

Universal Logic [10], proposed by $\mathrm{He}$ et al., is a kind of flexible logic. It considers the continuous change of not only the truth value of proposition, which is called truth value flexibility, but also the relation between propositions, which is called relational flexibility. Based on fuzzy logic, it puts up two important coefficients: generalized correlation coefficient " $h$ " and generalized self-correlation coefficient " $k$." The flexible change of universal logic operations is based on " $h$ " and " $k$." So, Universal Logic provides a new theoretical foundation to realize more accurate control for complex systems.

In our previous work [3], we focused on the basic physical meanings of fuzzy input variables of the normal twodimensional fuzzy controller, such as $E$ and EC. And we proved that the essential relation between them is just universal combinatorial relation in Universal Logic [10]. So, the simple universal combinatorial operation can be used instead of complex fuzzy reasoning process. As a result, a flexible logic control method was put forward.

Through the previous analysis, it is clear that flexible ability of flexible logic control model is resulted from the 
following aspect. We use universal combinatorial operation to reflect the essential relation between the deviation and the deviation change of control system, which considers the continuous change of the relation between things. And universal combinatorial operation model is not a single fixed operator, but a continuous cluster of combinatorial operators determined by the general correlation coefficient $h$ between propositions. In practical control application, according to the general correlation between propositions, we can take the corresponding one from the cluster to realize effective control for complex system.

However, in practical control application, how to determine the general correlation coefficient $h$ of flexible logic control model is a problem for further studies. First, the conventional universal combinatorial operation model has been limited in the interval $[0,1]$. Consequently, this paper studies a kind of universal combinatorial operation model based on the interval $[a, b]$. And some important theorems are given and proved, which provide a foundation for the control application of universal combinatorial operation. Then, this paper carries out the parametric analysis of flexible logic control model. And some research results have been given, which have important directive to determine the values of the general correlation coefficients in practical control application.

The rest of the paper is organized as follows. Section 2 introduces necessary background on universal combinatorial operation model and flexible logic control method and gives and proves some important theorems of universal combinatorial operation model based on the interval $[a, b]$. Section 3 carries out the parametric analysis of flexible logic control model and gives some research results. Finally, concluding remarks are given in Section 4.

\section{Universal Combinatorial Operation Model and Flexible Logic Control Method}

2.1. Universal Combinatorial Operation Model. It is well known that there is complex relation between every factor of complex system, which may be conflictive or consistent. For dealing reasonably with the complex relations, various aggregation operators have been given which are mostly $T$ norm, $S$-norm, or Mean operators. Nevertheless, $T$-norm, $S$ norm, and Mean operators have the following properties:

$$
\begin{gathered}
0 \leq T(x, y) \leq \min (x, y) \\
\max (x, y) \leq S(x, y) \leq 1 \\
\min (x, y) \leq M(x, y) \leq \max (x, y) .
\end{gathered}
$$

As a result, $T$-norm or $S$-norm can only handle mutually conflictive relation. In contrast, Mean operators can only handle mutually consistent relation. Therefore, the operating regions of these aggregation operators are localized. Universal combinatorial operation model is the combinatorial connective of Universal Logic, whose operating region is the standard interval $[0,1]$.
In the paper, we will only consider the generalized correlation coefficient $h$. So, zero-order universal combinatorial operation model is defined as follows.

Definition 1 (see [10]). Zero-order universal combinatorial operation model is the cluster

$$
\begin{aligned}
& C^{e}(x, y, h) \\
& =\text { ite }\left\{\Gamma^{e}\left[\left(x^{m}+y^{m}-e^{m}\right)^{1 / m}\right] \mid x+y<2 e ;\right. \\
& 1-\left(\Gamma ^ { 1 - e } \left[(1-x)^{m}+(1-y)^{m}\right.\right. \\
& \left.\left.\left.-(1-e)^{m}\right]\right)^{1 / m} \mid x+y>2 e ; e\right\},
\end{aligned}
$$

where $m=(3-4 h) /(4 h(1-h)), h \in[0,1], m \in R$, and $e \in$ $[0,1]$.

Remark 2. The conditional expression ite $\{\beta \mid \alpha ; \gamma\}$ represents that if $\alpha$ is true, then the result is $\beta$, otherwise $\gamma$. Similarly, $\operatorname{ite}\left\{\beta_{1}\left|\alpha_{1} ; \beta_{2}\right| \alpha_{2} ; \gamma\right\}=\operatorname{ite}\left\{\beta_{1} \mid \alpha_{1} ; \operatorname{ite}\left\{\beta_{2} \mid\right.\right.$ $\left.\alpha_{2} ; \gamma\right\}$. And $\Gamma^{1}[x]=\operatorname{ite}\{1|x>1 ; 0| x<$ 0 or $x$ is an imaginary number; $x\}$.

Universal combinatorial operation model is a continuous cluster of combinatorial operators, which can be continuously changeable with generalized correlation coefficient $h$ between propositions. In practical application, according to the general correlation between propositions, we can take the corresponding one from the cluster. As generalized correlation coefficient $h$ is equal to some special values, the corresponding combinatorial operators are given in Table 1.

2.2. Universal Combinatorial Operation Model on Any Interval $[a, b]$. In practical control application, fuzzy domain of fuzzy variables, $E$ and $E C$, is mostly symmetrical, such as $[-6,6]$. However, the conventional universal combinatorial operation model has been limited in the interval $[0,1]$. So, Chen [11] put forward a kind of universal combinatorial operation model, which is on any interval $[a, b]$.

Definition 3 (see [11]). Normal universal Not operation model in any interval $[a, b]$ is defined as follows:

$$
\mathrm{GN}(x)=b+a-x .
$$

For the previous definition, some common properties of normal universal Not operation model are given:

(1) closure

$$
\mathrm{GN}(x) \in[a, b],
$$

(2) two polar law

$$
\mathrm{GN}(a)=b, \quad \mathrm{GN}(b)=a,
$$

(3) symmetric involution

$$
\mathrm{GN}(\mathrm{GN}(x))=x \text {. }
$$


TABLE 1: Some special combinatorial operators of universal combinatorial operation model.

\begin{tabular}{|c|c|c|c|}
\hline Value of $h$ & $\begin{array}{l}\text { General correlation } \\
\text { between propositions }\end{array}$ & Combinatorial operator & Name of combinatorial operator \\
\hline 1 & Max-attracting & $\begin{array}{l}C^{e}(x, y, 1) \\
=\text { ite }\{\min (x, y) \mid x+y<2 e \\
\quad \max (x, y) \mid x+y>2 e \\
\quad e\}\end{array}$ & Zadeh combinatorial operator $C_{3}^{e}$ \\
\hline 0.75 & Independent correlation & $\begin{array}{l}C^{e}(x, y, 0.75) \\
=\operatorname{ite}\{x y / e \mid x+y<2 e \\
\quad(x+y-x y-e) /(1-e) \mid x+y>2 e \\
\quad e\}\end{array}$ & Probability combinatorial operator $C_{2}^{e}$ \\
\hline 0.5 & Max-rejecting & $C^{e}(x, y, 0.5)=\Gamma^{1}[x+y-e]$ & Bounded combinatorial operator $C_{1}^{e}$ \\
\hline 0 & Max-restraining & $\begin{array}{l}C^{e}(x, y, 0) \\
=\text { ite }\{0 \mid x, y<e \\
\quad 1 \mid x, y>e \\
\quad e\}\end{array}$ & Drastic combinatorial operator $C_{0}^{e}$ \\
\hline
\end{tabular}

Definition 4 (see [11]). Universal combinatorial operation model on any interval $[a, b]$ is the cluster

$$
\begin{aligned}
& \mathrm{GC}^{e}(x, y, h) \\
& =\text { ite }\{\min (e,(b-a) \\
& \times\left[\max \left(0, \frac{(x-a)^{m}+(y-a)^{m}-(e-a)^{m}}{(b-a)^{m}}\right)\right]^{1 / m} \\
& +a) \mid x+y<2 e \\
& b+a \\
& -\min \left(e^{\prime},(b-a)\right. \\
& \times\left[\max \left(0, \frac{(b-x)^{m}+(b-y)^{m}-(b-e)^{m}}{(b-a)^{m}}\right)\right]^{1 / m} \\
& +a) \mid x+y>2 e ; e\} \text {, }
\end{aligned}
$$

where $m=(3-4 h) /(4 h(1-h)), h \in[0,1], m \in R, e, e^{\prime} \in$ $[a, b]$, and $e^{\prime}=\mathrm{GN}(e)$.

Without loss of generality, we assume that the fuzzy domain of fuzzy variables, $E$ and $E C$, is the interval $[-1,1]$. As a result, when $a, b$, and $e$ are equal to $-1,1$, and 0 , respectively, the corresponding zero-order universal combinatorial operator is the cluster

$$
\begin{aligned}
& \operatorname{GC}^{0}(x, y, h) \\
& =\operatorname{ite}\{\min (0,2 \\
& \times\left[\max \left(0, \frac{(x+1)^{m}+(y+1)^{m}-1}{2^{m}}\right)\right]^{1 / m}
\end{aligned}
$$

$$
\begin{aligned}
& -1) \mid x+y<0 \\
& -\min \left(0,2\left[\max \left(0, \frac{(1-x)^{m}+(1-y)^{m}-1}{2^{m}}\right)\right]^{1 / m}\right. \\
& -1) \mid x+y>0 ; 0\} .
\end{aligned}
$$

According to the definition of Universal Combinatorial Operation Model in any interval, the following characters [11] are attained:

(1) $\mathrm{GC}^{e}(x, y, h)$ conforms to the combination axiom:

(a) boundary condition GC1

when $x, y<e, \operatorname{GC}^{e}(x, y, h) \leq \min (x, y)$; when $x, y>e, \operatorname{GC}^{e}(x, y, h) \geq \max (x, y)$; when $x+y=2 e, \mathrm{GC}^{e}(x, y, h)=e$; otherwise, $\min (x, y) \leq \mathrm{GC}^{e}(x, y, h) \leq$ $\max (x, y)$,

(b) monotonicity GC2

$\mathrm{GC}^{e}(x, y, h)$ increases monotonously along with $x$ and $y$,

(c) continuity GC3

when $h \in(0,1), \mathrm{GC}^{e}(x, y, h)$ is continuous for all $x$ and $y$,

(d) commutative law GC4

$$
\operatorname{GC}^{e}(x, y, h)=\operatorname{GC}^{e}(y, x, h),
$$

(e) law of identical element GC5

$$
\mathrm{GC}^{e}(x, e, h)=x,
$$

(2) closure

$$
\mathrm{GC}^{e}(x, y, h) \in[a, b],
$$


(3) inverse law

$$
\mathrm{GC}^{e}(x, 2 e-x, h)=e
$$

(4) renunciation law

$$
\operatorname{GC}^{e}(e, e, h)=e
$$

Theorem 5. Let $G N\left(G C^{G N(e)}(G N(x), G N(y), h)\right)=$ $G C^{e}(x, y, h), x, y \in[a, b], e \in[a, b]$.

Proof. $x, y \in[a, b], e \in[a, b]$, according to the closure of central generalized negation operation and universal combinatorial operation:

$\mathrm{GN}(x), \mathrm{GN}(y) \in[a, b], \mathrm{GN}(e) \in[a, b]$ and $\mathrm{GC}^{\mathrm{GN}(e)}(\mathrm{GN}(x), \mathrm{GN}(y), h) \in[a, b]$, and according to the definition of universal combinatorial operation:

(1) when $x+y<2 e$,

$$
\begin{aligned}
\mathrm{GN} & (x)+\mathrm{GN}(y) \\
& =(b+a-x)+(b+a-y) \\
& =2(b+a)-(x+y) \\
& >2(b+a-e)=2 \mathrm{GN}(e) .
\end{aligned}
$$

Then, according to the definition of $\mathrm{GC}^{e}(x, y, h)$,

$$
\begin{aligned}
& \mathrm{GC}^{\mathrm{GN}(e)}(\mathrm{GN}(x), \mathrm{GN}(y), h) \\
& =b+a-\min (b+a-\mathrm{GN}(e),(b-a) \\
& \times\left[\operatorname { m a x } \left(0, \frac{(b-\mathrm{GN}(x))^{m}}{(b-a)^{m}}\right.\right. \\
& +\frac{(b-\mathrm{GN}(y))^{m}}{(b-a)^{m}} \\
& \left.\left.\left.-\frac{(b-\mathrm{GN}(e))^{m}}{(b-a)^{m}}\right)\right]^{1 / m}+a\right) .
\end{aligned}
$$

According to the definition of central generalized negation operation,

$$
\begin{aligned}
& \mathrm{GN}(x)=b+a-x \\
& \mathrm{GN}(y)=b+a-y \\
& \mathrm{GN}(e)=b+a-e .
\end{aligned}
$$

Substituting (16), (17), and (18) separately into (15),

$$
\begin{aligned}
\mathrm{GC}^{\mathrm{GN}(e)}(\mathrm{GN}(x), \mathrm{GN}(y), h) & \\
=b+a-\min (e,(b-a) & \\
\times & {\left[\operatorname { m a x } \left(0, \frac{(x-a)^{m}+(y-a)^{m}}{(b-a)^{m}}\right.\right.} \\
& \left.\left.\left.-\frac{(e-a)^{m}}{(b-a)^{m}}\right)\right]^{1 / m}+a\right),
\end{aligned}
$$

and then

$$
\begin{aligned}
& \mathrm{GN}(\mathrm{GC}(e)(\mathrm{GN}(x), \mathrm{GN}(y), h)) \\
& =b+a-(b+a \\
& \quad-\min (e,(b-a) \\
& \times\left[\operatorname { m a x } \left(0, \frac{(x-a)^{m}}{(b-a)^{m}}\right.\right. \\
& \left.\left.+\frac{(y-a)^{m}-(e-a)^{m}}{(b-a)^{m}}\right)\right]^{1 / m} \\
& =\min (e,(b-a) \quad+a)) \\
& \quad \times\left[\operatorname { m a x } \left(0, \frac{(x-a)^{m}}{(b-a)^{m}}\right.\right. \\
& \left.\left.\left.+\frac{(y-a)^{m}-(e-a)^{m}}{(b-a)^{m}}\right)\right]^{1 / m}+a\right) . \\
& =\operatorname{GC}(x, y, h) .
\end{aligned}
$$

(2) When $x+y>2 e$,

$$
\begin{aligned}
\mathrm{GN}(x) & +\mathrm{GN}(y) \\
= & (b+a-x)+(b+a-y) \\
= & 2(b+a)-(x+y) \\
< & 2(b+a-e)=2 \mathrm{GN}(e) .
\end{aligned}
$$


So, according to the definition of $\mathrm{GC}^{e}(x, y, h)$,

$$
\begin{aligned}
& \mathrm{GC}^{\mathrm{GN}(e)}(\mathrm{GN}(x), \mathrm{GN}(y), h) \\
& =\min (\mathrm{GN}(e),(b-a) \\
& \times\left[\operatorname { m a x } \left(0, \frac{(\mathrm{GN}(x)-a)^{m}+(\mathrm{GN}(y)-a)^{m}}{(b-a)^{m}}\right.\right. \\
& \left.\left.\left.\quad-\frac{(\mathrm{GN}(e)-a)^{m}}{(b-a)^{m}}\right)\right]^{1 / m}+a\right) .
\end{aligned}
$$

Substituting (16), (17), and (18) separately into (22),

$$
\begin{aligned}
& \mathrm{GC}^{\mathrm{GN}(e)}(\mathrm{GN}(x), \mathrm{GN}(y), h) \\
& =\min (b+a-e,(b-a) \\
& \times\left[\operatorname { m a x } \left(0, \frac{(b-x)^{m}}{(b-a)^{m}}\right.\right. \\
& \left.\left.\left.+\frac{(b-y)^{m}-(b-e)^{m}}{(b-a)^{m}}\right)\right]^{1 / m}+a\right) .
\end{aligned}
$$

And then,

$$
\begin{aligned}
& \mathrm{GN}\left(\mathrm{GC}^{\mathrm{GN}(e)}(\mathrm{GN}(x), \mathrm{GN}(y), h)\right) \\
& =b+a-\min (b+a-e,(b-a) \\
& \left.\times\left[\max \left(0, \frac{(b-x)^{m}}{(b-a)^{m}}\right)\right]^{1 / m}+a\right) \\
& \left.+\frac{(b-y)^{m}-(b-e)^{m}}{(b-a)^{m}}\right) \\
& =\mathrm{GC}^{e}(x, y, h) .
\end{aligned}
$$

(3) When $x+y=2 e$,

$$
\begin{aligned}
\mathrm{GN} & (x)+\mathrm{GN}(y) \\
& =(b+a-x)+(b+a-y) \\
& =2(b+a)-(x+y) \\
& =2(b+a-e) \\
& =2 \mathrm{GN}(e) .
\end{aligned}
$$

According to the definition of $\mathrm{GC}^{e}(x, y, h)$,

$$
\mathrm{GC}^{\mathrm{GN}(e)}(\mathrm{GN}(x), \mathrm{GN}(y), h)=\mathrm{GN}(e) .
$$

Then,

$$
\mathrm{GN}\left(\mathrm{GC}^{\mathrm{GN}(e)}(\mathrm{GN}(x), \mathrm{GN}(y), h)\right)=\mathrm{GN}(\mathrm{GN}(e))=e \text {. }
$$

From the aforementioned, the theorem is true.

Lemma 6. Let $G C^{G N(e)}(G N(x), G N(y), h)=G N\left(G C^{e}(x, y\right.$, h)).

Proof. According to Theorem 5 and involution law of central generalized negation operator in interval $[a, b]$, the theorem can be proved simply.

Lemma 7. Let $C^{e}(x, y, h)=1-C^{1-e}(1-x, 1-y, h)$.

Proof. Setting the interval $[a, b]$ of $x, y$ as $[0,1]$, the lemma can be proved simply.

Lemma 8. When interval $[a, b]$ relates to e symmetry, $G C^{e}\left(x^{*}, y^{*}, h\right)=\left(G C^{e}(x, y, h)\right)^{*}$, wherein $x^{*}$ represents the points of $x$ relating to e symmetry, namely, $x^{*}=2 e-x, y^{*}$, $e^{*},\left(G C^{e}(x, y, h)\right)^{*}$ is similar, $e \in[a, b], h \in[0,1]$.

Proof. Since interval $[a, b]$ relates to $e$ symmetry, then $a+b=$ $2 e$, and $\mathrm{GN}(x)=a+b-x=2 e-x$. Thus,

$$
\mathrm{GN}(x)=x^{*}
$$

Similarly,

$$
\begin{gathered}
\mathrm{GN}(y)=y^{*}, \\
\mathrm{GN}(e)=a+b-e=2 e-e=e .
\end{gathered}
$$

From (28) and (29), the following could be obtained:

$$
\begin{gathered}
\operatorname{GC}^{\mathrm{GN}(e)}(\mathrm{GN}(x), \mathrm{GN}(y), h)=\mathrm{GC}^{e}\left(x^{*}, y^{*}, h\right), \\
\mathrm{GN}\left(\mathrm{GC}^{e}(x, y, h)\right)=\left(\operatorname{GC}^{e}(x, y, h)\right)^{*} .
\end{gathered}
$$

And then from Lemma 6,

$$
\mathrm{GC}^{e}\left(x^{*}, y^{*}, h\right)=\left(\mathrm{GC}^{e}(x, y, h)\right)^{*} .
$$

So, the theorem is true.

Lemma 9. Let $C^{0.5}(1-x, 1-y, h)=1-C^{0.5}(x, y, h)$, wherein $h \in[0,1]$.

Lemma 10. When interval $[a, b]$ relates to symmetry of original point, $C^{0}(-x,-y, h)=-G C^{0}(x, y, h), h \in[0,1]$.

This lemma indicates that when the interval $[a, b]$ is symmetrical about the origin point and identity element $e$ 
is 0, Universal Combinatorial Operation $\mathrm{GC}^{e}(x, y, h)$ is also symmetrical about the origin point.

As pointed out in the literature [3], the internal relation between fuzzy input variables, the deviation $E$, and the deviation change $E C$ of normal two-dimensional fuzzy controller is the universal combination relation in universal logic. Consequently, the complex fuzzy rule inference process could be replaced by the simple universal combinatorial operation, and a flexible logic control model was presented accordingly. In fuzzy control, the domain of input variables and output variable is generally symmetrical about the original point, such as $[-5,5]$. Obviously, it is the prerequisite of control model that the operation model relates to symmetry of original point. Therefore, Lemma 10 provides a basis for the Universal Combinatorial Operation's application in control.

2.3. Universal Combinatorial Operation Model with Unequal Weights. In practical complex system, every factor is generally with unequal weight. But the existing universal combinatorial operation only discusses an ideal state that every factor is with equal weight.

2.3.1. Weighted Operator. For dealing reasonably with the complex relations of every factor in complex system, various properties which weighted operators should have are put forward. The weighted operator proposed by Yager is one of the famous ones.

The weighted operator proposed by Yager is defined as follows.

Definition 11 (see [12]). Assume that an operator $h(\alpha, x)$ is a mapping from $[0,1]$ to $[0,1]$.

$h(\alpha, x)$ is called a Yager weighted operator if it satisfies the following properties.

(I1) Monotonicity with respect to the value, $x$. In particular if $x>x^{\prime}$, then we require

$$
h(\alpha, x) \geq h\left(\alpha, x^{\prime}\right) .
$$

(I2) We desire that elements with weight zero have no effect on the aggregation process; thus, if $e$ is the fixed identity of the aggregation to be used on the resulting bag, we must have

$$
h(0, x)=e .
$$

(I3) A normalcy with respect to the weights

$$
h(1, x)=x .
$$

(I4) Finally, we desire that the transformation moves monotonically from its value for $\alpha=0$ to $\alpha=1$. That is, if $x \geq e, h(\alpha, x)$ increases monotonically with respect to the value $\alpha$; if $x \leq e, h(\alpha, x)$ decreases monotonically with respect to the value $\alpha$,

where $\alpha$ is the weight associated with an argument, and $x$ is the argument. Both $\alpha$ and $x$ are drawn from $[0,1]$.

And due to the definition of Yager weighted operator, we can draw the following theorems.

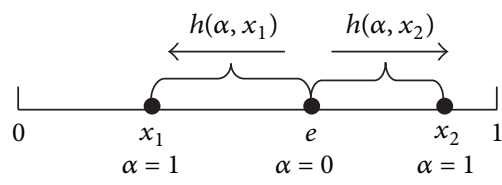

FIgURE 1: Yager weighted operator $h(\alpha, x)$ varies with the weight $\alpha$.

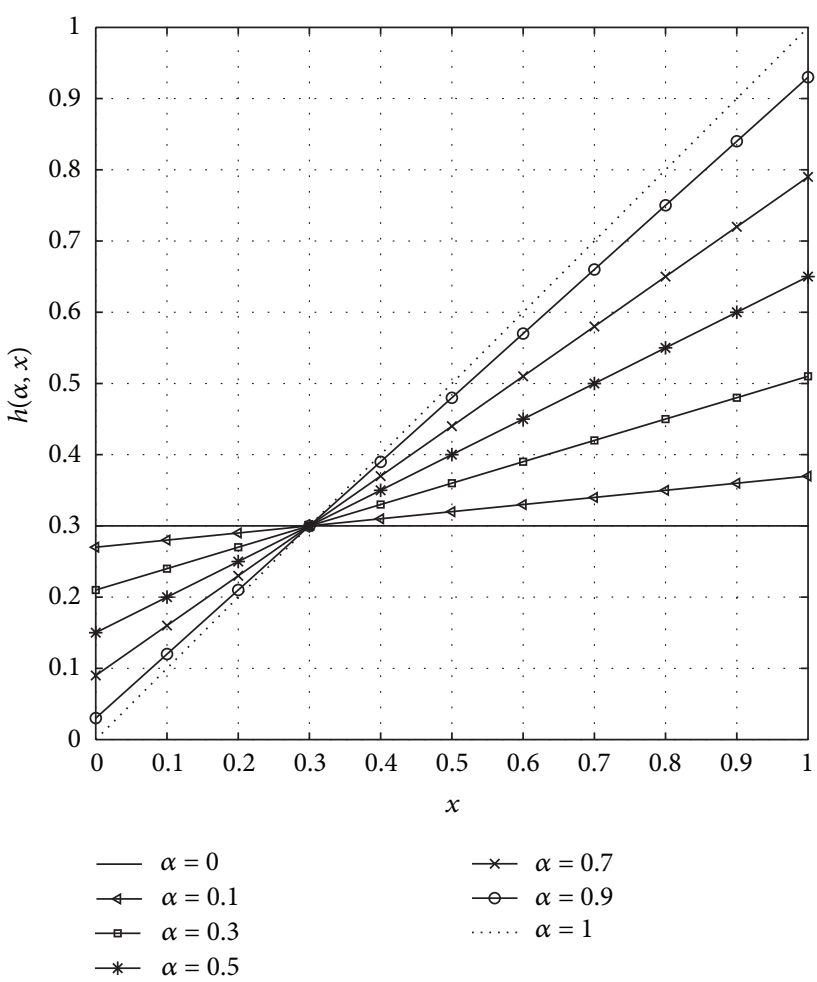

FIGURE 2: Yager weighted operator $h(\alpha, x)=\alpha x+(1-\alpha) e$ with $e$ is equal to 0.3 .

Theorem 12. Assume that $h(\alpha, x)$ is a Yager weighted operator. So, one has the following:

$$
\text { if } x \geq e \text {, then } h(\alpha, x) \geq e \text {, and if } x \leq e \text {, then } h(\alpha, x) \leq e \text {. }
$$

Proof. Due to the properties (I2) and (I4) of the definition, the theorem can be proved easily.

Theorem 13. Assume that $h(\alpha, x)$ is a Yager weighted operator. So, one has the following:

$$
\text { if } x \geq e \text {, then } h(\alpha, x) \geq e \text {, and if } x \leq e \text {, then } h(\alpha, x) \leq e \text {. }
$$

Proof. Due to the properties (I3) and (I4) of the definition, the theorem can be proved easily.

The chart of Yager weighted operator $h(\alpha, x)$ changing along with the weight $\alpha$ is given in Figure 1 .

One formulation that satisfies these conditions is $h(\alpha, x)=\alpha x+(1-\alpha) e$. It is described by Figure 2 .

According to the previous analyses, we discover that Yager weighted operator has some shortcomings as follows. 


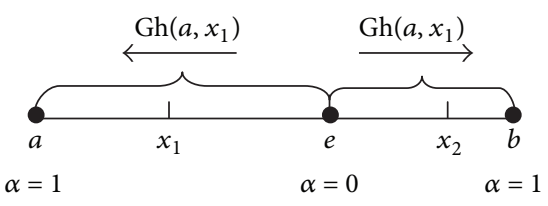

FIGURE 3: General weighted operator varies with the weight $\alpha$.

(1) Yager weighted operator is likely to transform entire True (False) proposition into partial True (False) proposition. However, due to the view of logic, entire True (False) proposition should still be transformed into entire True (False) proposition by weighted operator.

(2) The weighted value changes in the interval $[e, x]$ or $[x, e]$ for any weight $\alpha \in[0,1]$ as shown in Figure 1 . However, the weighted value is desired to change in the total interval $[0,1]$ in some practical applications.

(3) Yager weighted operator is limited in the interval $[0,1]$. But weighted operators are desired to change in the general interval $[a, b]$ in some practical applications.

For solving the previous problems, the paper puts forward a kind of general weighted operators $\operatorname{Gh}(\alpha, x)$, which change in the general interval $[a, b]$.

Definition 14. Assume that an operator $\operatorname{Gh}(\alpha, x)$ is a mapping from $[a, b]$ to $[a, b] . \mathrm{Gh}(\alpha, x)$ is called a general weighted operator if it satisfies the following properties.

(I1) Monotonicity with respect to the value, $x$. In particular, if $x>x^{\prime}$, then we require

$$
\mathrm{Gh}(\alpha, x) \geq \mathrm{Gh}\left(\alpha, x^{\prime}\right)
$$

(I2) $\operatorname{Gh}(0, x)=\operatorname{ite}\{a|x=a ; b| x=b ; e\}$.

(I3) $\mathrm{Gh}(\alpha, e)=e$.

(I4) $\operatorname{Gh}(\alpha, a)=a$ and $\operatorname{Gh}(\alpha, b)=b$.

(I5) Finally, we desire that the transformation moves monotonically for the weight $\alpha \in(0,1)$. That is, if $x \geq e, \operatorname{Gh}(\alpha, x)$ increases monotonically with respect to the value $\alpha$; if $x \leq e, \mathrm{Gh}(\alpha, x)$ decreases monotonically with respect to the value $\alpha$.

(I6) $\mathrm{Gh}(1, x)=\operatorname{ite}\{e|x=e ; a| x<e ; b \mid x>e\}$.

$\alpha$ is the weight associated with an argument $x$, and $e$ is the fixed identity of the aggregation operator $\mathrm{GC}^{e} . \alpha$ is drawn from $[0,1]$, and $e$ is drawn from $[a, b]$.

According to the previous definition, we can know that the absolute value of the argument $x$ decreases first and then increases with the weight $\alpha$ changing continuously from 0 to 1. The chart of general weighted operator changing along with the weight $\alpha$ is given in Figure 3 .

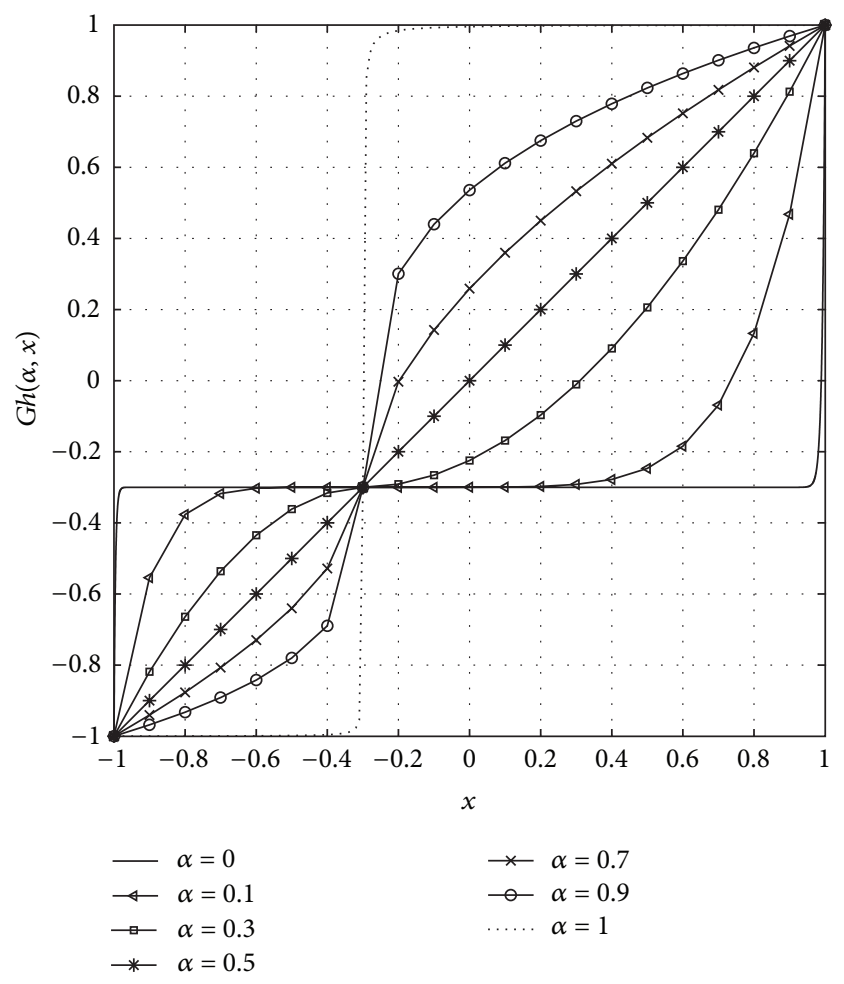

FIGURE 4: General weighted operator of polynomial model with $a=$ $-1, b=1$, and $e=-0.3$.

The common general weighted operation model is polynomial model:

$$
\begin{aligned}
& \text { Gh }(\alpha, x) \\
& =\operatorname{ite}\left\{\frac{(x-e)(b-e)(1+n)^{1 / 2}}{\left((b-x)+(1+n)^{1 / 2}(x-e)\right)}+e \mid x>e ;\right. \\
& \\
& \left.e-\frac{(e-x)(e-a)(1+n)^{1 / 2}}{\left((x-a)+(1+n)^{1 / 2}(e-x)\right)} \mid x<e ; e\right\} .
\end{aligned}
$$

Assume that $n=(2 \alpha-1) /(1-\alpha)^{2}, \alpha \in[0,1], n \geq-1$, and $\mathrm{Gh}(\alpha, x)$ is the limit as $\alpha=0$ and $\alpha=1$. So, (38) describes a general weighted operator as shown in Figure 4.

2.3.2. Universal Combinatorial Operation Model with Unequal Weights. According to the previous definition of general weighted operators, we can get the definition of universal combinatorial operation model with unequal weights as follows.

Definition 15. Assume that an operator $\operatorname{UGC}^{e}\left(x, y, \alpha_{x}, \alpha_{y}, h\right)$ is a mapping from $[a, b]$ to $[a, b] \cdot \operatorname{UGC}^{e}\left(x, y, \alpha_{x}, \alpha_{y}, h\right)$ is called universal combinatorial operation model with unequal weights:

$$
\operatorname{UGC}^{e}\left(x, y, \alpha_{x}, \alpha_{y}, h\right)=\operatorname{GC}^{e}\left(\operatorname{Gh}\left(\alpha_{x}, x\right), \operatorname{Gh}\left(\alpha_{y}, y\right), h\right),
$$




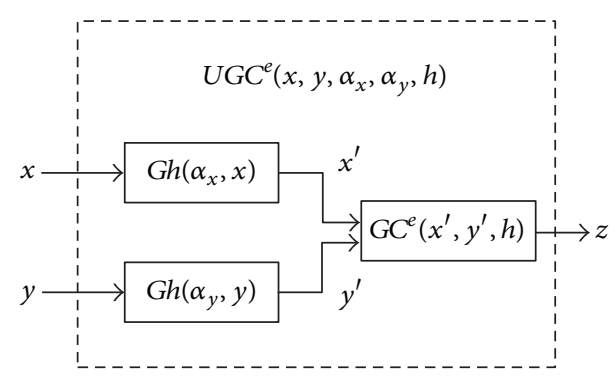

FIGURE 5: Universal combinatorial operation model with unequal weights.

where $\mathrm{Gh}(\alpha, x)$ is general weighted operator, $\mathrm{GC}^{e}(x, y, h)$ is universal combinatorial operator, $e$ is the fixed identity of $\mathrm{GC}^{e}(x, y, h), h$ is the generalized correlation coefficient, and $\alpha_{x}$ and $\alpha_{y}$ denote, respectively, the weights associated with the arguments $x$ and $y . x, y$, and $e$ are drawn from $[a, b]$, and $h, \alpha_{x}$, and $\alpha_{y}$ are drawn from $[0,1]$.

The universal combinatorial operation model with unequal weights is given in Figure 5.

2.4. Flexible Logic Control Method. In order to decrease effectively the number of fuzzy control rules for multivariable nonlinear system, Xiao et al. [13] gave a new concept of fuzzy composed variable. Its basic idea can be summarized as follows. According to characteristics of controlled system and internal relations between input variables, a fuzzy composed variable is constructed to reflect synthetically the deviation between reference and the process output with a fuzzy logic system.

There are four output variables in single inverted-pendulum, which are $x, x^{\prime}, \theta$, and $\theta^{\prime}$. In the four input variables of control system, it is $\theta$ and $\theta^{\prime}$ that describe the movement state of the rod. So, a fuzzy logic system can be designed, which is described by the fuzzy rules in Table 2, to define a fuzzy composed variable $G E_{\theta}$ with $\theta$ and $\theta^{\prime}$. The fuzzy composed variable $G E_{\theta}$ can describe synthetically the movement state of the rod. Similarly, a fuzzy composed variable $G E_{x}$ can be defined with $x$ and $x$ ' to describe synthetically the movement state of the cart. For multivariable system, one does not need to define, respectively, fuzzy logic system for every fuzzy composed variable. A uniform fuzzy rule table can be used, such as Table 2, but only select different quantification factors to obtain different fuzzy composed variables.

Remark 16. In the paper, the input variables of the fuzzy controllers discussed are $e$ and $e c$, which denote, respectively, the deviation and the deviation change. The output variable $u$ is the control signal. The variables, $e, e c$, and $u$ are crisp values from the practical process. The fuzzy variables, $E, E C$, and $U$ are the corresponding fuzzy ones, and the fuzzy domains are uniformed to be $[-1,1]$ with fuzzy subsets, such as NB, NM, NS, ZE, PS, PM, and PB.

Apparently, the fuzzy rules in Table 2 describe the essential relation between all deviation and the deviation
TABLE 2: Fuzzy rules defining fuzzy composed variable $G E_{\theta}$.

\begin{tabular}{|c|c|c|c|c|c|c|c|c|}
\hline \multirow{2}{*}{\multicolumn{2}{|c|}{$G E_{\theta}$}} & \multicolumn{7}{|c|}{$\theta^{\prime}$} \\
\hline & & NB & NM & NS & $\mathrm{ZE}$ & PS & PM & $\mathrm{PB}$ \\
\hline & $\mathrm{NB}$ & $\overline{\mathrm{NB}}$ & $\overline{\mathrm{NB}}$ & $\overline{\mathrm{N}} \overline{\mathrm{B}}$ & $\mathrm{NM}$ & $\overline{\mathrm{N}}$ & $\overline{\mathrm{N}} \overline{\mathrm{S}}$ & $\overline{\mathrm{ZE}_{\mathrm{I}}^{\prime}}$ \\
\hline & NM & : & NB & $\mathrm{NM}_{1}^{\prime}$ & NM & NS & ZE & PS \\
\hline & NS & NBB & NM & NM & NS & ZE & PS & $\mathrm{PM}_{1}^{\prime}$ \\
\hline \multirow[t]{4}{*}{$\theta$} & $\mathrm{ZE}$ & NM & NM & NS & ZE & PS & PM & $\mathrm{PM}$ \\
\hline & PS & $i^{\prime N} \bar{M}$ & $\overline{\mathrm{N}} \overline{\mathrm{s}}$ & $\overline{\mathrm{ZE}}$ & PS & $\begin{array}{c}\bar{P} \\
\end{array}$ & $\overline{\mathrm{PM}}$ & $\overline{\mathrm{P}} \overline{\mathrm{B}}$ \\
\hline & PM & NS & ZE & PS ! & PM & $\mathrm{PM}$ & PB & $\mathrm{PB}$ \\
\hline & $\mathrm{PB}$ & ZE & PS & $\mathrm{PM}^{\prime}$ & PM & $\mathrm{PB}$ & $\mathrm{PB}$ & $\mathrm{PB}$ \\
\hline
\end{tabular}

change. As shown Table 2 can be divided approximately into four parts, which describe, respectively, fuzzy rules used to define composed variable as $E$ and $E C$ are both negative, $E$ is negative but $E C$ is positive, $E$ and $E C$ are both positive, and $E$ is positive but $E C$ is negative. $E$ and $E C$ both describe the deviation between the reference and the process output; so, we can define a composed variable, denoted as $E^{\prime}$, based on the essential relation between them.

According to the physical meanings of fuzzy variables, $E$ and $E C$, we can get the following conclusions.

(1) Suppose that both $E$ and EC are positive. That is to say, the deviation is positive and it will increase continuously. So, the value of the composed variable $E^{\prime}$ should be positive and bigger than both of $E$ and $E C$ in this case. The combinatorial rules are shown by the right-bottom part of Table 2.

(2) Suppose that $E$ is positive and $E C$ is negative. That is to say, the deviation is positive but it will decrease. So, the value of the composed variable $E^{\prime}$ should be between $E$ and $E C$ in this case. The combinatorial rules are shown by the left-bottom part of Table 2 .

(3) Similarly, we can obtain the value of $E^{\prime}$ in the two of other cases.

Based on the previous analysis, we get the conclusion that the essential relation among $E, E C$, and the composed variable $E^{\prime}$ is a kind of universal combinatorial one in Universal Logic. As a result, we have

$$
E^{\prime}=\mathrm{GC}^{e}(E, E C, h) .
$$

So, we can obtain the relation among $E, E C$, and the output variable $U$ of control system as follows:

$$
U=-\mathrm{GC}^{e}(E, E C, h),
$$

where fuzzy variables, $E, E C, U \in[-1,1], e=0, h \in[0,1]$. $\mathrm{Fu}$ and $\mathrm{He}$ [3] named the method Flexible Logic Control Method. 


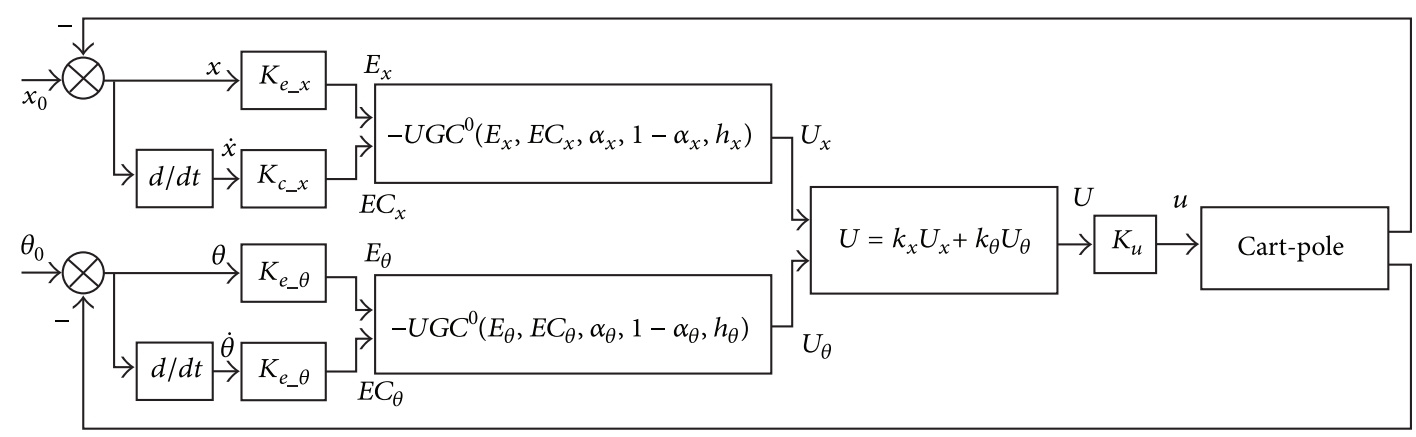

FIgURE 6: The flexible logic control model of single inverted-pendulum.

For effectively controlling different things, we can lead into a weighted factor $\alpha, \alpha \in[0,1]$. As the correlation coefficient $h$ is equal to 0.5 , we have

$$
\begin{aligned}
U & =-\mathrm{GC}^{e}(\alpha E,(1-\alpha) E C, 0.5) \\
& =\Gamma_{-1}^{1}[\alpha E+(1-\alpha) E C-e] \\
& =\alpha E+(1-\alpha) E C .
\end{aligned}
$$

However, (42) is just the fuzzy control method proposed by Long et al. [14]. They used a linear equation, such as (42), to describe fuzzy control rules. But the relation among $E, E C$, and $U$ is not only linear. So, (41) is a cluster of operators determined by correlation coefficient $h$, and (42) is only a special operator in the cluster as $h$ is equal to 0.5 . As a result, flexible logic control method can realize the accurate control for complex system.

\subsection{Flexible Logic Control Model of Single Inverted-Pendulum} System. The objective is to maintain the pole in an upright position and the cart in an appointed position in the rail. There are four output variables and one input variable in single inverted-pendulum, which are $x, x^{\prime}, \theta, \theta^{\prime}$, and $u$. In the four input variables of control system, both $\theta$ and $\theta^{\prime}$ describe the movement state of the pole, and both $x$ and $x^{\prime}$ describe the movement state of the cart. So, we have designed two subcontrollers. One is to maintain the cart in an appointed position with two input variables $E_{x}$ and $E C_{x}$. The other one is to maintain the pole in an upright position with two input variables $E_{\theta}$ and $E C_{\theta}$.

We have designed the two subcontrollers with the flexible logic control method. And we led into weighted factors $\alpha_{\theta}$, $\alpha_{x}, \alpha_{x}, \alpha_{\theta} \in[0,1]$. Two controllers are designed as follows:

$$
\begin{gathered}
U_{\theta}=-\mathrm{GC}^{0}\left(\mathrm{Gh}\left(\alpha_{\theta}, E_{\theta}\right), \mathrm{Gh}\left(1-\alpha_{\theta}, E C_{\theta}\right), h_{\theta}\right), \\
\alpha_{\theta}=\left(\alpha_{s_{-} \theta}-\alpha_{0 \_}\right)\left|E_{\theta}\right|+\alpha_{0 \_}, \\
U_{x}=-\mathrm{GC}^{0}\left(\mathrm{Gh}\left(\alpha_{x}, E_{x}\right), \mathrm{Gh}\left(1-\alpha_{x}, E C_{x}\right), h_{x}\right), \\
\alpha_{x}=\left(\alpha_{s_{-} x}-\alpha_{0 \_x}\right)\left|E_{x}\right|+\alpha_{0 \_x} .
\end{gathered}
$$

When the control signal $U$ is combined, we lead into two weighted factors $k_{\theta}, k_{x}, k_{\theta}, k_{x} \in[-1,1]$, for the two subcontrollers. According to (43) and (45), we can get the output of the controller as follows:

$$
\begin{aligned}
U= & k_{x} U_{x}+k_{\theta} U_{\theta} \\
= & k_{x}\left(-\operatorname{GC}^{0}\left(\mathrm{Gh}\left(\alpha_{x}, E_{x}\right), \operatorname{Gh}\left(1-\alpha_{x}, E C_{x}\right), h_{x}\right)\right) \\
& +k_{\theta}\left(-\mathrm{GC}^{0}\left(\mathrm{Gh}\left(\alpha_{\theta}, E_{\theta}\right), \mathrm{Gh}\left(1-\alpha_{\theta}, E C_{\theta}\right), h_{\theta}\right)\right),
\end{aligned}
$$

where $E_{x}, E C_{x}, U_{x}, E_{\theta}, E C_{\theta}, U_{\theta}, U \in[-1,1], \operatorname{Gh}(\alpha, x)(38)$, $\operatorname{UGC}^{0}\left(x, y, \alpha_{1}, \alpha_{2}, h\right)$ is (39), $h_{x}, h_{\theta}$ are general correlation coefficients, $h_{x}, h_{\theta} \in[0,1], \alpha_{x}, \alpha_{\theta} \in[0,1], 0 \leq \alpha_{0 \_} \leq \alpha_{s_{-} x} \leq$ 1 , and $0 \leq \alpha_{0 \_} \leq \alpha_{s \_\theta} \leq 1$.

By the previous analyses, we can obtain the control model of single inverted-pendulum as shown in Figure 6.

\section{Parametric Analysis of Flexible Logic Control Model}

Through the previous analysis, it is clear that flexible ability of flexible logic control model is resulted from the following aspect. Universal combinatorial operation model is not a single fixed operator, but a continuous cluster of combinatorial operators determined by the general correlation coefficient $h$ between propositions. In practical control application, according to the general correlation between propositions, we can take the corresponding one from the cluster to realize effective control for complex system.

However, in practical control application, how to determine the general correlation coefficient $h$ of flexible logic control model is a problem for further studies. In this section, we will analyze the general correlation coefficients, $h_{x}, h_{\theta}$, of the flexible logic control model and give some research results.

3.1. Experimentation. We experiment the flexible logic control model in some single inverted-pendulum physical system. The physical parameters of the system are given in Table 3.

By simulating the inverted-pendulum and looking up the optimization with genetic algorithm, we can get the initial values of the control parameters. Then, by testing in real-time experimentations and repeatedly making some fine tuning, 
TABLE 3: Physical parameters of the quadruple inverted-pendulum.

\begin{tabular}{|c|c|c|}
\hline Symbol & Value & Meaning \\
\hline$m_{0}$ & $0.924 \mathrm{~kg}$ & Mass of the cart \\
\hline$m_{1}$ & See Table 5 & Mass of the pole \\
\hline$f_{0}$ & $0.1 \mathrm{~N} \cdot \mathrm{s} / \mathrm{m}$ & $\begin{array}{l}\text { Dynamic friction coefficient between the } \\
\text { cart and the track }\end{array}$ \\
\hline$f_{1}$ & $0.007056 \mathrm{~N} \cdot \mathrm{s} / \mathrm{m}$ & Dynamic friction coefficient for the pole \\
\hline$l_{1}$ & See Table 5 & $\begin{array}{l}\text { Distance from the position sensor to the } \\
\text { center of gravity of the pole }\end{array}$ \\
\hline
\end{tabular}

TABLE 4: Control parameters of the control model.

\begin{tabular}{|c|c|c|}
\hline Symbol & Value & Meaning \\
\hline$K_{e \_x}$ & 23.5294 & Quantification factor for $E_{x}$ \\
\hline$K_{c \_x}$ & 9.4118 & Quantification factor for $E C_{x}$ \\
\hline$\alpha_{0-x}$ & 0.1725 & Minimum value of $\alpha_{x}$ \\
\hline$\alpha_{s_{-} x}$ & 0.4683 & Maximum value of $\alpha_{x}$ \\
\hline$K_{e_{-} \theta}$ & 50.5882 & Quantification factor for $E_{\theta}$ \\
\hline$K_{c_{-} \theta}$ & 3.1765 & Quantification factor for $E C_{\theta}$ \\
\hline$\alpha_{0 \_} \theta$ & 0.2510 & Minimum value of $\alpha_{\theta}$ \\
\hline$\alpha_{s_{-} \theta}$ & 0.5953 & Maximum value of $\alpha_{\theta}$ \\
\hline$k_{x}$ & -0.2235 & Weighted factor of the subcontroller for the cart \\
\hline$k_{\theta}$ & 0.4902 & Weighted factor of the subcontroller for the pole \\
\hline$K_{u}$ & 8.7843 & Proportion factor for $U$ \\
\hline$h_{x}$ & 0.2118 & $\begin{array}{l}\text { General correlation coefficient between } E_{x} \text { and } \\
E C_{x}\end{array}$ \\
\hline$h_{\theta}$ & 0.9490 & $\begin{array}{l}\text { General correlation coefficient between } E_{\theta} \text { and } \\
E C_{\theta}\end{array}$ \\
\hline
\end{tabular}

TABLE 5: The variation of the length of the pole.

\begin{tabular}{lcc}
\hline No. & $m_{1}(\mathrm{~kg})$ & $L_{1}(\mathrm{~m})$ \\
\hline 1 & 0.0149 & 0.1 \\
2 & 0.0216 & 0.149 \\
3 & 0.0284 & 0.199 \\
4 & 0.0378 & 0.266 \\
5 & 0.0493 & 0.354 \\
6 & 0.0621 & 0.4436 \\
7 & 0.0773 & 0.553 \\
8 & 0.0966 & 0.691 \\
\hline
\end{tabular}

we can get the control parameters shown in Table 4. And the variation of the length of the pole is shown in Table 5.

Genetic algorithm is used to optimize the general correlation coefficients, $h_{x}, h_{\theta}$, and the fitness function is defined as follows:

$$
\begin{gathered}
\text { Dis }=\sum_{i=0}^{N} \frac{i^{2} \times\left(x^{2}(i) / 10+x^{\prime 2}(i) / 20+\theta^{2}(i)+\theta^{\prime 2}(i)\right)}{N}, \\
\text { fitness }=\frac{1}{(\text { Dis } / 10)} .
\end{gathered}
$$

TABLE 6: The optimization results of $h_{x}, h_{\theta}$.

\begin{tabular}{lccccc}
\hline No. & $m_{1}(\mathrm{~kg})$ & $L_{1}(\mathrm{~m})$ & $h_{x}$ & $h_{\theta}$ & Fitness \\
\hline 1 & 0.0149 & 0.1 & 0.985 & 0.845 & 0.4310 \\
2 & 0.0216 & 0.149 & 0.975 & 0.845 & 0.3310 \\
3 & 0.0284 & 0.199 & 0.96 & 0.845 & 0.2713 \\
4 & 0.0378 & 0.266 & 0.955 & 0.87 & 0.2240 \\
5 & 0.0493 & 0.354 & 0.63 & 0.88 & 0.1838 \\
6 & 0.0621 & 0.4436 & 0.37 & 0.875 & 0.1531 \\
7 & 0.0773 & 0.553 & 0.32 & 0.865 & 0.1260 \\
8 & 0.0966 & 0.691 & 0.37 & 0.85 & 0.1029 \\
\hline
\end{tabular}

Through simulated optimization, we can get the corresponding values of $h_{x}, h_{\theta}$ and the fitness shown in Table 6.

Figure 7 shows how the fitness varies with $h_{x}, h_{\theta}$. And Figures 8 and 9 illustrate how the maximum fitness varies with $h_{\theta}$ taking different values when $h_{x}$, in turn, is equal to some value on standard interval $[0,1]$. Figure 8 shows how the maximum fitness varies with $h_{x}$, and Figure 9 shows how $h_{\theta}$ varies with $h_{x}$ when the fitness is the maximum.

Similarly, Figures 10 and 11 illustrate how the maximum fitness varies with $h_{x}$ taking different values when $h_{\theta}$, in turn, is equal to some value on standard interval $[0,1]$. Figure 10 shows how the maximum fitness varies with $h_{\theta}$, and Figure 11 shows how $h_{x}$ varies with $h_{\theta}$ when the fitness is the maximum.

3.2. Parametric Analysis of Flexible Logic Control Model. Firstly, calculate the correlation coefficient between fitness and $h_{x}$ when $h_{\theta}$, in turn, is equal to some value on standard interval $[0,1]$. The formula for calculating the correlation coefficient is defined as follows:

$$
r=\frac{\sum_{m} \sum_{n}\left(A_{m n}-\bar{A}\right)\left(B_{m n}-\bar{B}\right)}{\sqrt{\left(\sum_{m} \sum_{n}\left(A_{m n}-\bar{A}\right)^{2}\right)\left(\sum_{m} \sum_{n}\left(B_{m n}-\bar{B}\right)^{2}\right)}},
$$

where $A$ and $B$ are $m * n$ matrices, $\bar{A}$ and $\bar{B}$ are the means of the values in $A$ and $B$, respectively, and $r$ is the correlation coefficient between $A$ and $B$.

Figure 12 illustrates how the correlation coefficient between fitness and $h_{x}$ varies with $h_{\theta}$ taking different values on standard interval $[0,1]$.

Similarly, calculate the correlation coefficient between fitness and $h_{\theta}$ when $h_{x}$, in turn, is equal to some value on standard interval $[0,1]$. And Figure 13 illustrates how the correlation coefficient between fitness and $h_{\theta}$ varies with $h_{x}$ taking different values on standard interval $[0,1]$.

After that, the correlation coefficients between the maximum fitness and $h_{x}, h_{\theta}$ are computed for poles of different lengths, as shown in Table 7.

By observing and analyzing the previous results, we can draw the following conclusions.

(1) The longer the pole, the worse the control effects. Figures 7, 8, and 10 all indicate that the longer the pole, the smaller the fitness with the optimum control parameters, and vice versa. Through experiment, we can know that the given single inverted-pendulum 


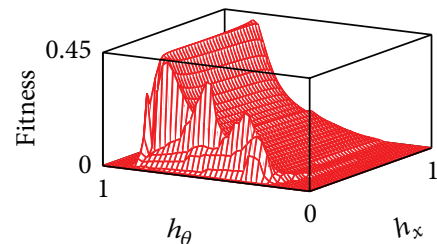

(a)

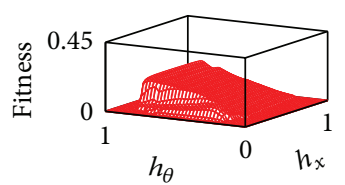

(e)

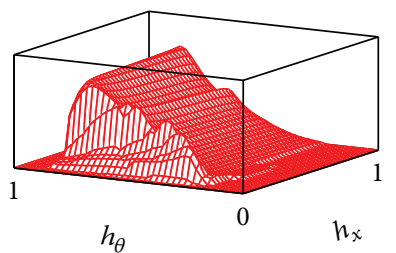

(b)

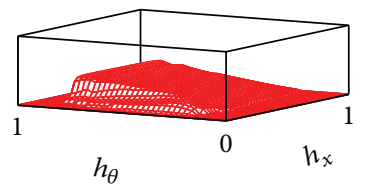

(f)

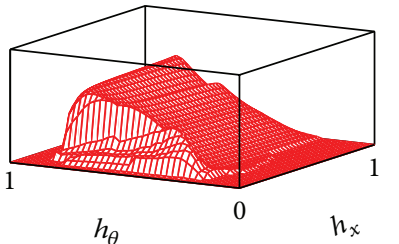

(c)

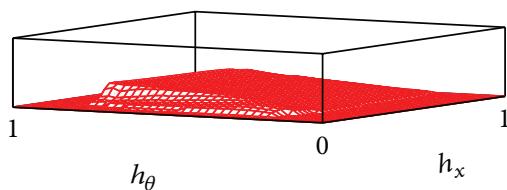

(g)

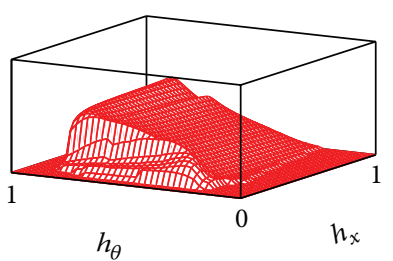

(d)

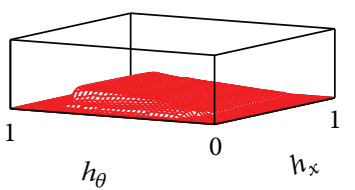

(h)

FIGURE 7: The fitness varies with $h_{x}, h_{\theta}$.

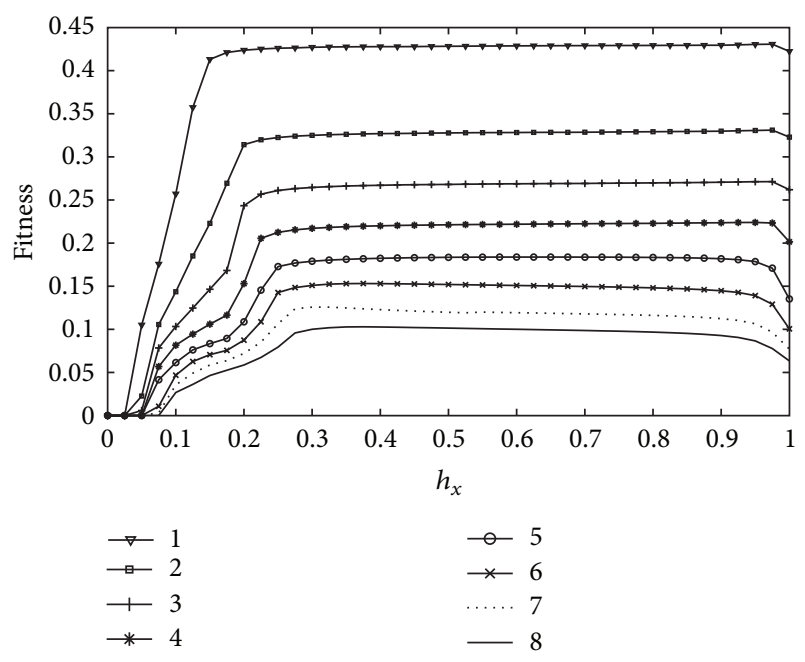

FIGURE 8: The maximum fitness varies with $h_{x}$.

physical system can be controlled effectively when the pole is in the range $0.1 \mathrm{~m}$ to $0.691 \mathrm{~m}$. That is to say, when the pole is longer than $0.691 \mathrm{~m}$ or shorter than $0.1 \mathrm{~m}$, we cannot realize the stable control for the given physical system.

(2) For some given physical system, the control effect is sensitive to the value of $h_{\theta}$. This means that we can realize the effective control only when $h_{\theta}$ is in some very short interval. And the interval is relatively fixed. That is, the interval of $h_{\theta}$ does not change with the length of the pole. Figures 7, 9 and 10 all show that the fitness is relatively big only when $h_{\theta}$ is in the range 0.8 to 0.9 , and the interval of $h_{\theta}$ remains unchanged for poles of different lengths.

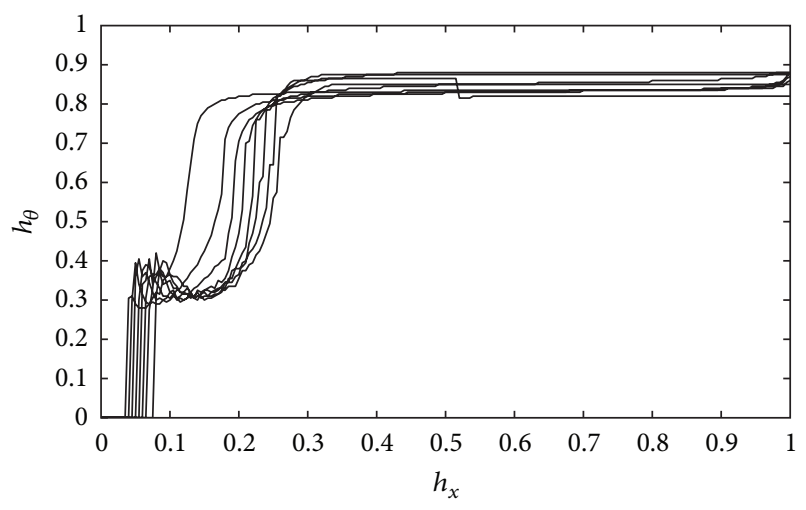

FIGURE 9: $h_{\theta}$ varies with $h_{x}$ when the fitness is the maximum.

Figure 12 also shows that the correlation coefficient between fitness and $h_{x}$ is relatively big only when $h_{\theta}$ is around 0.2 or in the range 0.8 to 0.9 , and the interval of $h_{\theta}$ remains unchanged for poles of different lengths.

(3) For some given physical system, the control effect is not sensitive to the value of $h_{x}$. That is, we can realize the effective control when $h_{x}$ is in some very long interval. Figure 10 shows that the control effect does not change much with $h_{x}$ taking different values of the long interval.

Figure 13 also shows that the correlation coefficient between fitness and $h_{\theta}$ is relatively big when $h_{x}$ is in the range 0.15 to 0.98 .

However, the width of the interval varies with the length of pole. The longer the pole, the narrower the interval. The paper calls the interval of $h_{x}$ as $h_{x}$ platform.

(4) For poles of different lengths, there is much difference of $h_{x}$ and little one of $h_{\theta}$ when we realize the most effective control. As shown in Table 7, the correlation 

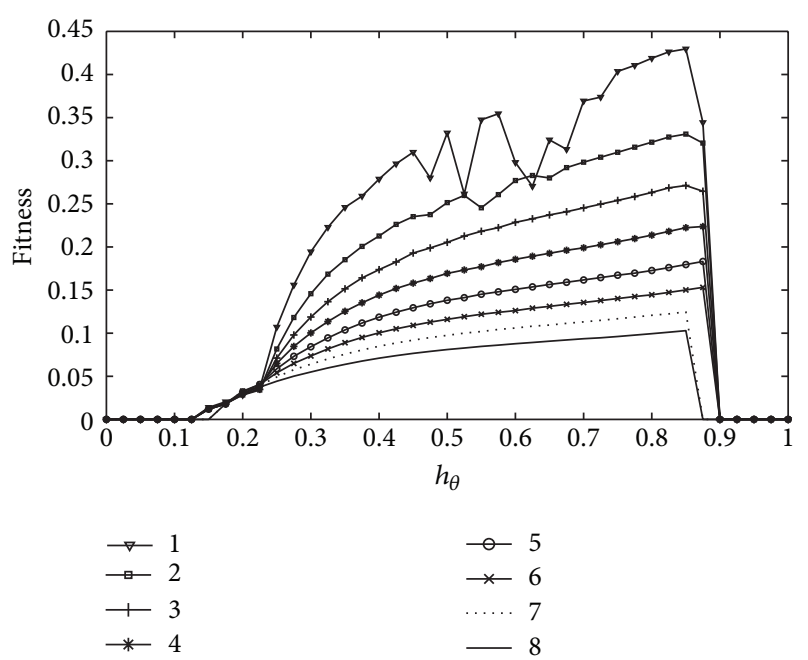

Figure 10: The maximum fitness varies with $h_{\theta}$.

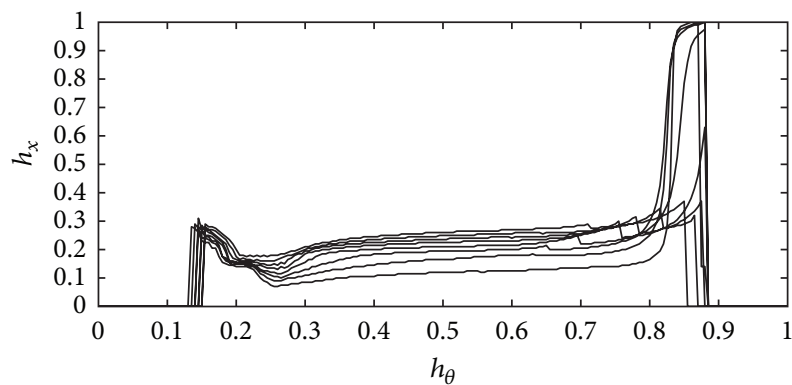

FIGURE 11: $h_{x}$ varies with $h_{\theta}$ when the fitness is the maximum.

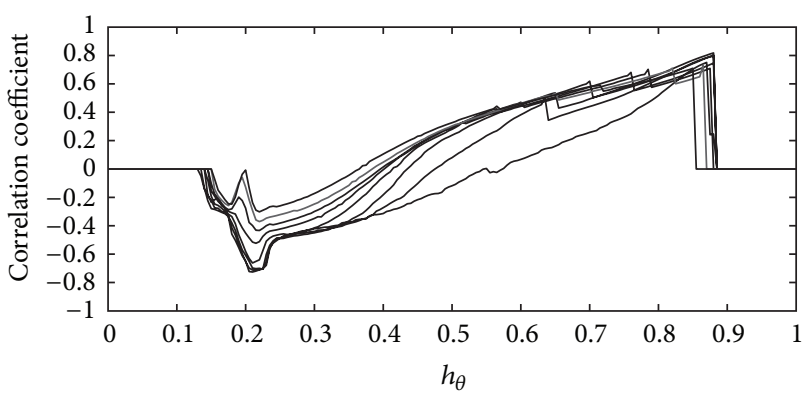

FIGURE 12: The correlation coefficient between fitness and $h_{x}$ varies with $h_{\theta}$.

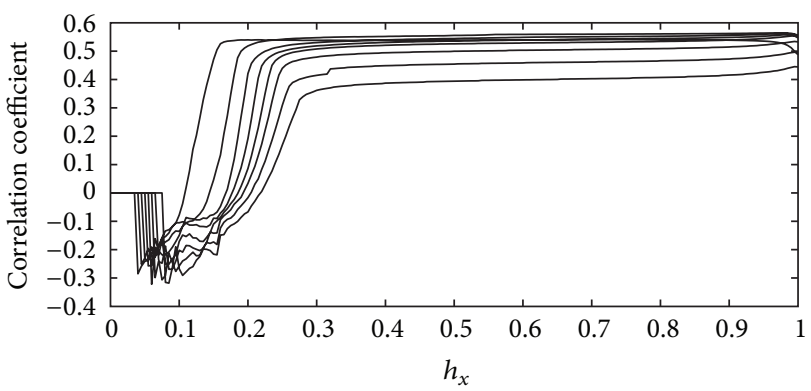

FigURE 13: The correlation coefficient between fitness and $h_{\theta}$ varies with $h_{x}$.

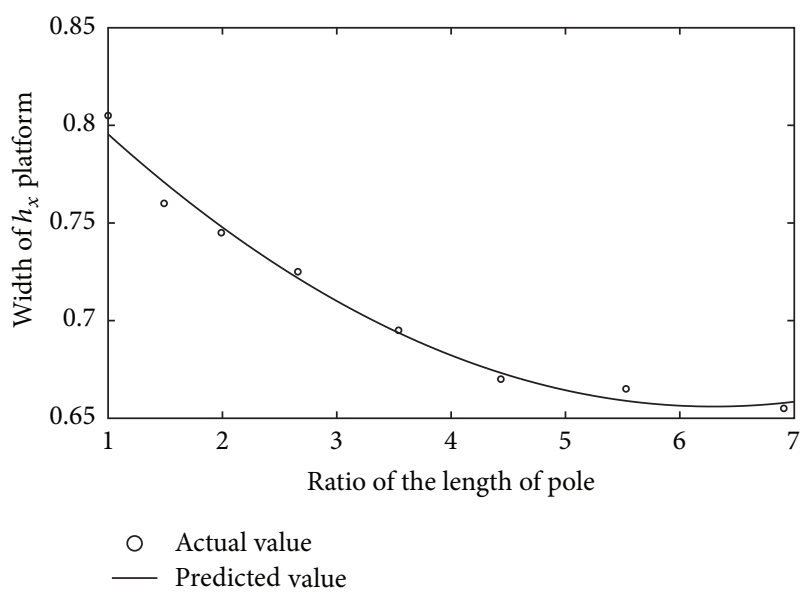

FIgURE 14: The width of $h_{x}$ platform varies with the ratio of the length.

TABLE 7: The correlation coefficients between the maximum fitness and $h_{x}, h_{\theta}$.

\begin{tabular}{ccccc}
\hline No. $m_{1}(\mathrm{~kg})$ & $L_{1}(\mathrm{~m}) \begin{array}{c}\text { The correlation } \\
\text { coefficient between } \\
\text { the maximum } \\
\text { fitness and } h_{x}\end{array}$ & $\begin{array}{c}\text { The correlation } \\
\text { coefficient between } \\
\text { the maximum } \\
\text { fitness and } h_{\theta}\end{array}$ \\
\hline 1 & 0.0149 & 0.1 & 0.3801 & 0.9602 \\
2 & 0.0216 & 0.149 & 0.3956 & 0.9503 \\
3 & 0.0284 & 0.199 & 0.4104 & 0.9537 \\
4 & 0.0378 & 0.266 & 0.4261 & 0.9604 \\
5 & 0.0493 & 0.354 & 0.4285 & 0.9513 \\
6 & 0.0621 & 0.4436 & 0.4294 & 0.9314 \\
7 & 0.0773 & 0.553 & 0.4308 & 0.9125 \\
8 & 0.0966 & 0.691 & 0.4327 & 0.8895 \\
\hline
\end{tabular}

coefficient between the maximum fitness and $h_{x}$ is small, but the one between the maximum fitness and $h_{\theta}$ is big. Table 6 shows that the longer the pole, the smaller the optimum $h_{x}$, and the optimum $h_{\theta}$ is in the range 0.8 to 0.9 with little difference.

From the third conclusion, we can know that the width of $h_{x}$ platform varies with the length of pole. That is, the longer the pole, the smaller the width. For poles of different lengths, the corresponding $h_{x}$ platforms are shown in Table 8. And the relation between the width of $h_{x}$ platform and the length of pole and the one between the starting value of $h_{x}$ platform and the length of pole can be obtained through fitting method according to the experimental results shown in Table 8.

Firstly, suppose that the smallest effective length of pole, that is, $0.1 \mathrm{~m}$, is 1 unit. Then, the ratios of the other lengths to the smallest effective length of pole are shown in Table 8. The relation between the width of $h_{x}$ platform and the ratio of the length of pole is depicted by means of the linear fit, which is as shown in (50) and Figure 14. Consider

$$
y=0.85314-0.062624 x+0.0049723 x^{2} .
$$


TABLE 8: The corresponding $h_{x}$ platforms for poles of different lengths.

\begin{tabular}{lccccc}
\hline No. & $L_{1}(\mathrm{~m})$ & Ratio of the lengths of poles & $\begin{array}{c}\text { The starting value } \\
\text { of } h_{x} \text { platform }\end{array}$ & $\begin{array}{c}\text { The finishing value } \\
\text { of } h_{x} \text { platform }\end{array}$ & $\begin{array}{c}\text { The width } \\
\text { of } h_{x} \text { platform }\end{array}$ \\
\hline 1 & 0.1 & 1 & 0.175 & 0.98 & 0.905 \\
2 & 0.149 & 1.49 & 0.22 & 0.98 & 0.76 \\
3 & 0.199 & 1.99 & 0.235 & 0.98 & 0.745 \\
4 & 0.266 & 2.66 & 0.25 & 0.975 & 0.725 \\
5 & 0.354 & 3.54 & 0.26 & 0.94 & 0.695 \\
6 & 0.4436 & 4.436 & 0.27 & 0.94 & 0.67 \\
7 & 0.553 & 5.53 & 0.275 & 0.94 & 0.655 \\
8 & 0.691 & 6.91 & 0.285 & & \\
\hline
\end{tabular}

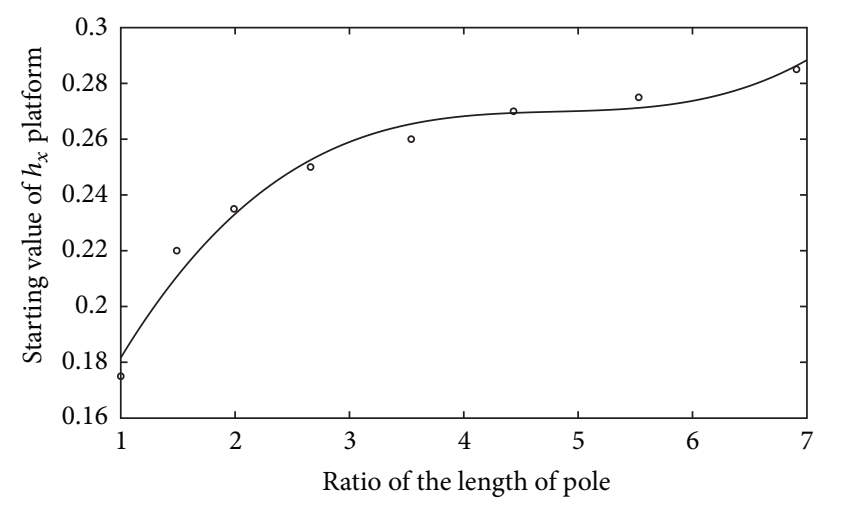

○ Actual value

— Predicted value

FIGURE 15: The starting value of $h_{x}$ platform varies with the ratio of the length.

Similarly, the relation between the starting value of $h_{x}$ platform and the ratio of the length of pole is depicted by means of the linear fit, which is as shown in (51) and Figure 15. Consider

$$
y=0.095132+0.10701 x-0.022054 x^{2}+0.0015301 x^{3} .
$$

Hence, for the given physical system, when the length of pole takes different values in the previous controllable interval, we can calculate the corresponding interval of $h_{x}$ platform by (50) and (51). Then, $h_{x}$ can be any of the interval and $h_{\theta}$ should be any of the interval $[0.8,0.9]$. Therefore, it does not need to optimize the control parameters again, and we can realize the effective control for the physical system with new length of pole.

\section{Conclusion}

Flexible logic method uses universal combinatorial operation model to describe the logic relation between $E$ and $E C$, which are the fuzzy input variables of normal two-dimensional fuzzy controller. Universal combinatorial operation model is not a single fixed operator, but a continuous cluster of combinatorial operators determined by the general correlation coefficient $h$ between propositions. In practical control application, according to the general correlation between propositions, we can take the corresponding one from the cluster to realize effective control for complex system.

However, in practical control application, how to determine the general correlation coefficient $h$ of flexible logic control model is a problem for further studies. First, the conventional universal combinatorial operation model has been limited in the interval $[0,1]$. Consequently, this paper studies a kind of universal combinatorial operation model based on any interval $[a, b]$. And some important theorems are given and proved, which provide a foundation for the flexible logic control method. For dealing reasonably with the complex relations of every factor in complex system, a kind of universal combinatorial operation model with unequal weights is put forward. Then, this paper has carried out the parametric analysis of flexible logic control model. And some research results have been given, which have important directive to determine the values of the general correlation coefficients in practical control application.

\section{Acknowledgments}

This work is supported by the Beijing Municipal Natural Science Foundation (nos. 4113069 and 4122007), the Major State Basic Research Development Program of China (no. 2007CB311100), and the Beijing Municipal Education Commission Foundation (no. 007000546311501).

\section{References}

[1] L. M. Hou, H. G. Zhang, X. C. Liu, E. H. Chu, and Q. Wang, "Adaptive fuzzy sliding mode soft switch of speed sensorless for PMSM based on robust passivity-based control," Control and Decision, vol. 25, no. 5, pp. 686-690, 2010.

[2] H. X. Li, "To see the success of fuzzy logic from mathematical essence of fuzzy control," Fuzzy Systems and Mathematics, vol. 9, no. 4, pp. 1-14, 1995.

[3] L. H Fu and H. C. He, "Studies on control method based on flexible logic," Computer Science, vol. 36, no. 2, 2009.

[4] H. R. Dong, B. Gao, B. Ning, and Y. X. Zhang, "Fuzzy-PID soft switching speed control of automatic train operation system," Control and Decision, vol. 25, no. 5, pp. 794-800, 2010. 
[5] G. Sun and W. Huo, "Direct-adaptive fuzzy predictive control of satellite attitude," Acta Automatica Sinica, vol. 36, no. 8, pp. 1151-1159, 2010.

[6] N. B. He, Q. Gao, C. S. Jiang, and C. L. Gong, "Adaptive fuzzy control for MIMO non-affine nonlinear systems," Control Theory and Applications, vol. 27, no. 12, pp. 1783-1786, 2010.

[7] M. G. Joo and T. Sudkamp, "A method of converting a fuzzy system to a two-layered hierarchical fuzzy system and its runtime efficiency," IEEE Transactions on Fuzzy Systems, vol. 17, no. 1, pp. 93-103, 2009.

[8] J. F. Qiao and H. D. Wang, "Structure self-organizing algorithm for fuzzy neural networks and its applications," Control Theory and Applications, vol. 25, no. 4, pp. 703-707, 2008.

[9] H. X. Li, "Adaptive fuzzy controllers based on variable universe," Science in China. Series E. Technological Sciences, vol. 42, no. 1, pp. 10-20, 1999.

[10] H. C. He, H. Wang, Y. H. Liu, Y. J. Wang, and Y. W. Du, Universal Logics Principle, Science Press, Marrickville, Australia, 2001.

[11] Z. C. Chen, Studies on Correlation Reasoning of Fractal, Chaos, and Logic in Complex System[D], Northwestern Polytechnical University, Xi'an, China, 2004.

[12] R. R. Yager and A. Rybalov, "Full reinforcement operators in aggregation techniques," IEEE Transactions on Systems, Man, and Cybernetics, Part B: Cybernetics, vol. 28, no. 6, pp. 757-769, 1998.

[13] J. Xiao, S. Zhang, and X. H. Xu, "Self-adaptive weighted control based on fuzzy composed variables," Control and Decision, vol. 16, no. 2, pp. 191-194, 2001.

[14] S. Z. Long and P. Z. Wang, "The self-adjustment of fuzzy control rules," Fuzzy Mathematics, vol. 3, no. 3, pp. 105-112, 1982. 


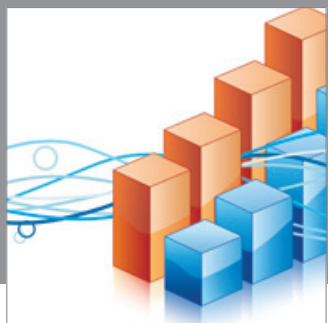

Advances in

Operations Research

mansans

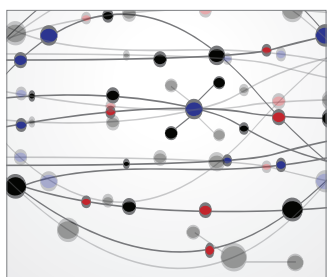

The Scientific World Journal
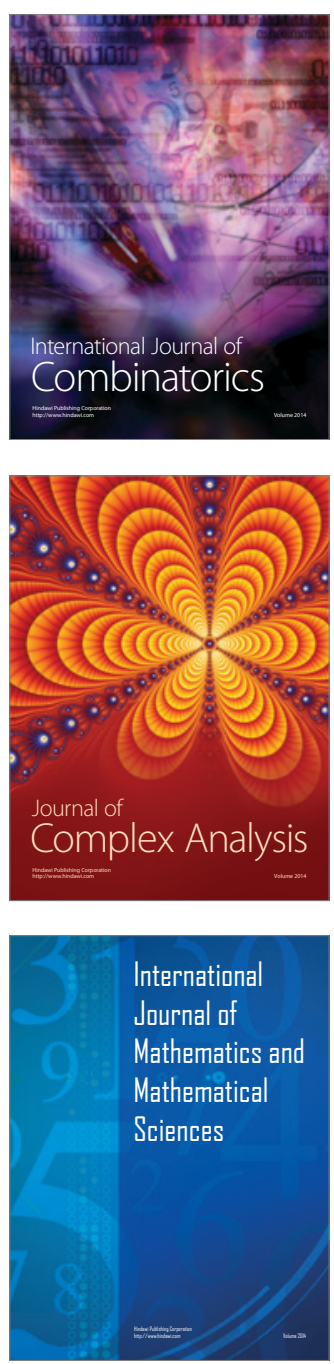
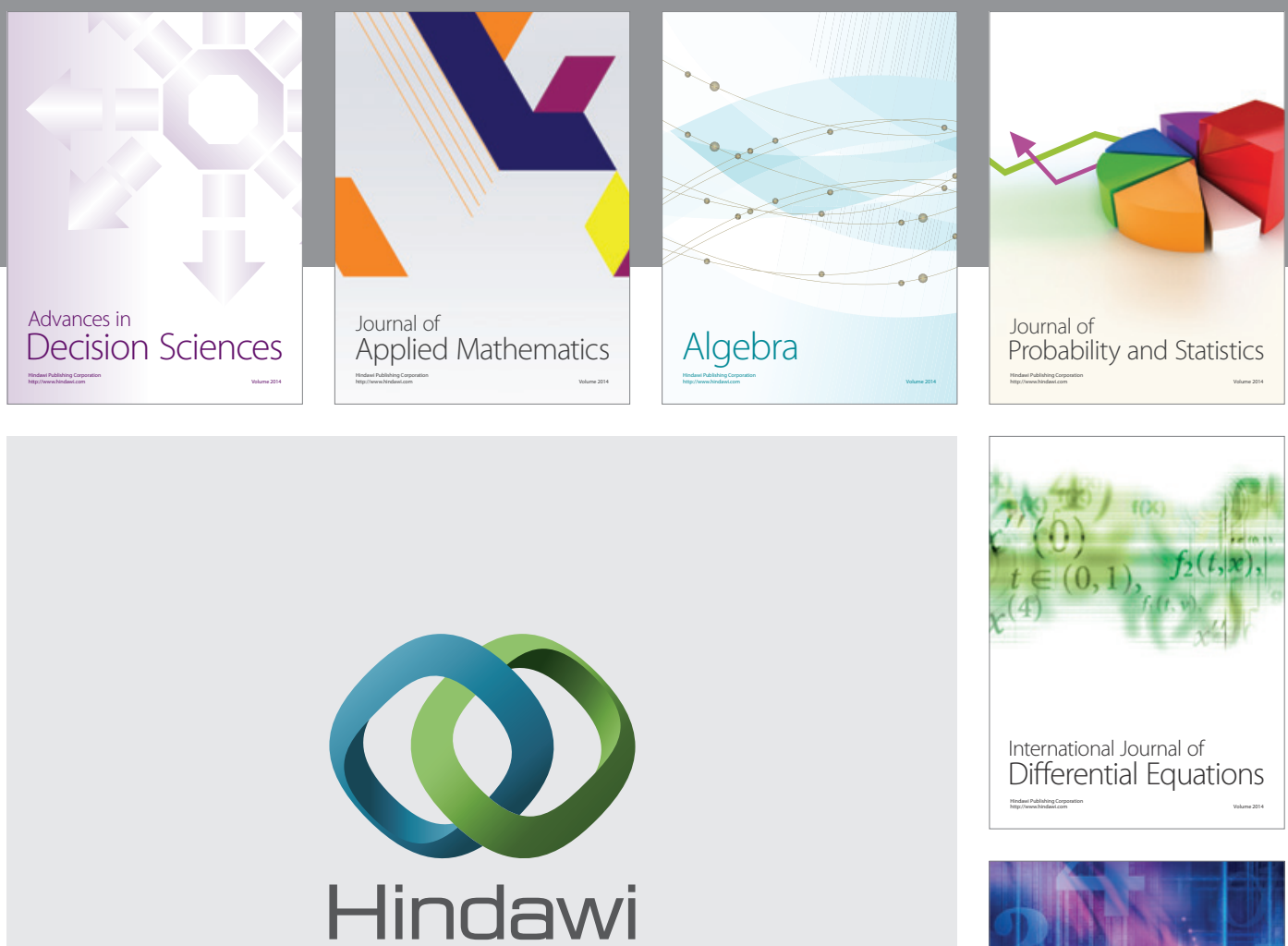

Submit your manuscripts at http://www.hindawi.com
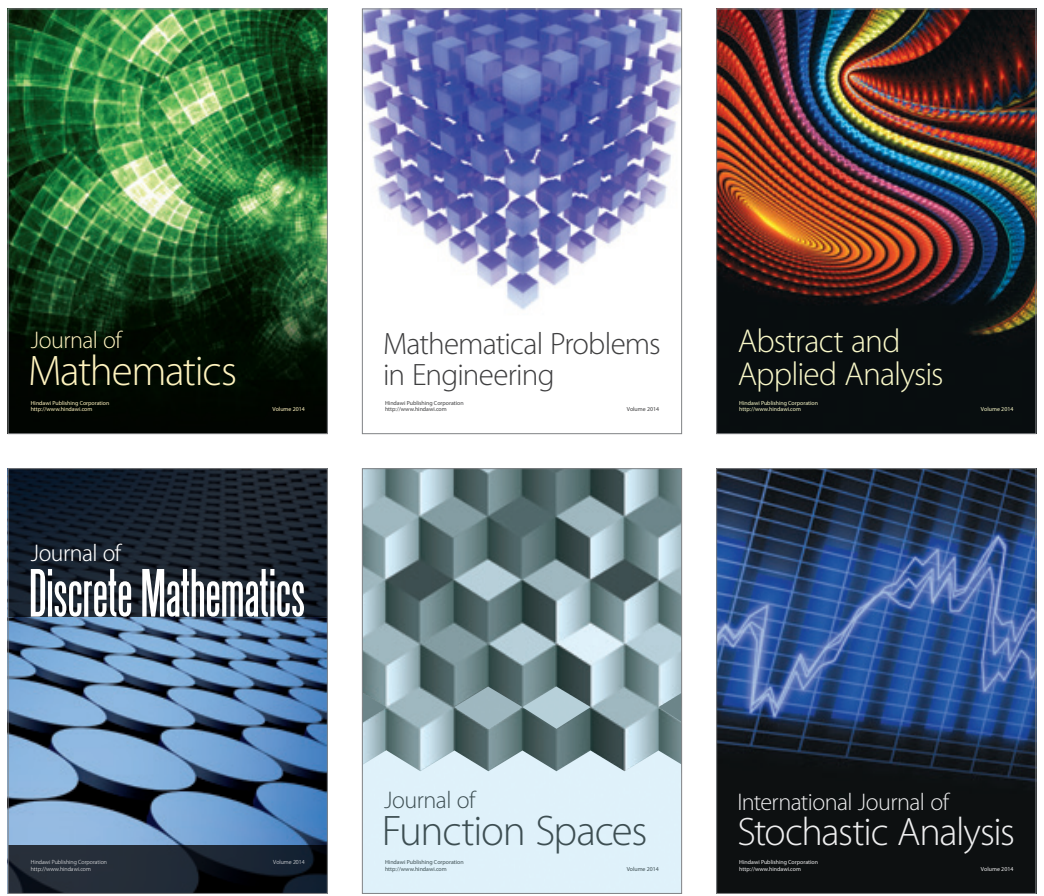

Journal of

Function Spaces

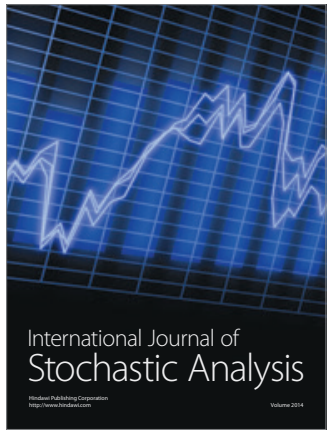

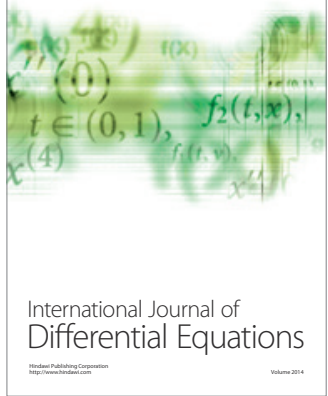
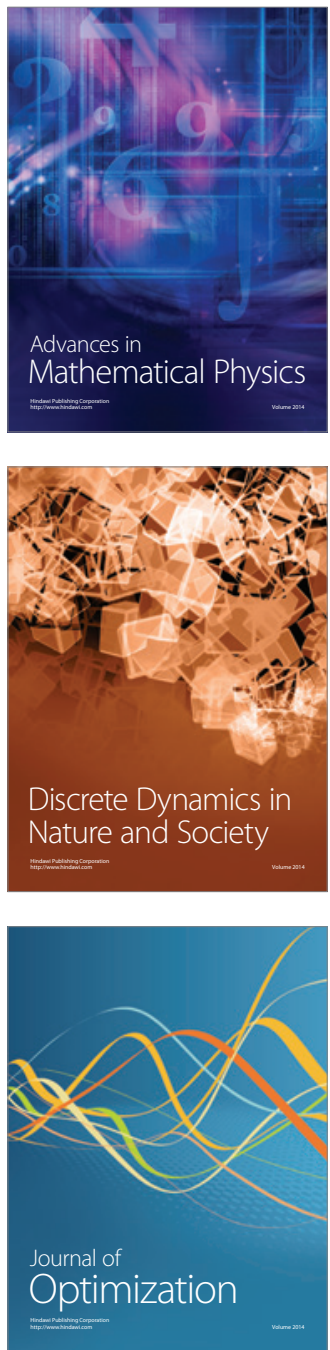